\title{
A Fluorescent Sensor for Imaging Reversible Redox Cycles in Living Cells
}

\author{
Evan W. Miller, Shelly X. Bian, and Christopher J. Chang \\ Department of Chemistry, University of California, Berkeley, California 94720
}

Life in aerobic environments requires organisms to maintain strict control over their internal redox status. At the cellular level, aerobic respiration poses a particularly unique challenge for living systems, as the energy-releasing reduction of oxygen to water generates partially reduced reactive oxygen species (ROS) intermediates that can exert widely divergent physiological and/or pathological effects. ${ }^{1}$ Unregulated production of ROS results in oxidative stress, and subsequent buildup of free radical damage to proteins, lipids, and nucleic acids is connected to serious human diseases where age is a risk factor. ${ }^{2,3}$ However, emerging evidence suggests that controlled production of one ROS in particular, hydrogen peroxide, can mediate cellular signal transduction through reversible oxidation and reduction of cysteine thiols and other redox-active groups. ${ }^{4-8}$

The complex, reversible oxidation biology of the cell and its broad implications in human health and disease provide motivation for developing new ways to study dynamic redox chemistry in living systems. In this regard, fluorescence imaging with redox-responsive chemosensors is a potentially powerful approach to probe various stages of oxidative signaling, stress, or repair in real time in living cells. Traditional fluorescent probes for redox activity, including dichlorodihydrofluorescein or dihydrorhodamine 123, are useful for cellular studies but can only respond irreversibly to a single initial oxidation event. ${ }^{9-11}$ In contrast, fluorophores that can respond reversibly to changes in oxidation or reduction events would be much more valuable for visualizing cycles of redox signaling, stress, or repair and their dynamic interconversion. A few redox-sensitive fluorescent reporters based on protein ${ }^{12,13}$ or peptide ${ }^{14,15}$ scaffolds have been described, but no small molecules have been reported to date for imaging reversible oxidation and reduction events in living biological systems. We now present the synthesis, properties, and live-cell imaging applications of Redoxfluor-1 (RF1), a new type of fluorescent sensor for detecting reversible redox cycles in aqueous solution and in living cells. RF1 features a dual colorimetric/fluorimetric readout for oxidation-reduction events, a >50-fold fluorescence dynamic range, and visible wavelength excitation and emission profiles to minimize cellular damage and autofluorescence. In addition, RF1 can be loaded into living cells and image multiple, reversible cycles of oxidative stress and reductive repair.

Our design strategy for fluorescence detection of reversible oxidation-reduction events is inspired by the extensive use of disulfides as redox resevoirs in biology. We anticipated that integrating this unit into a fluorescein scaffold would provide a small-molecule redox reporter with desirable optical properties and biological compatibility. Along these lines, the synthesis of RF1 proceeds in three steps as shown in Scheme 1. Lithiation of naphthalene in the presence of TMEDA and quenching with sulfur affords disulfide $\mathbf{1}$ according to literature procedures. 16 Vilsmeier formylation of $\mathbf{1}$ with $\mathrm{POCl}_{3} / \mathrm{DMF}$ generates 2- and 4-formyl isomers that are

chrischang@berkeley.edu.

Supporting Information Available: Synthetic and experimental details (PDF). This material is available free of charge via the Internet at http://pubs.acs.org. 
separable by flash column chromatography in $54 \%$ (2a) and $26 \%$ (2b) yields, respectively. Acid-catalyzed condensation of $\mathbf{2 b}$ with 2 equiv of resorcinol furnishes RF1 in $12 \%$ yield.

Spectroscopic experiments with RF1 were performed under simulated physiological conditions (20 mM HEPES, pH 7). As expected, the oxidized probe displays fluorescein-like characteristics with a strong absorption band centered at $490 \mathrm{~nm}\left(\varepsilon=4.0 \times 10^{4} \mathrm{M}^{-1} \mathrm{~cm}^{-1}\right)$ and bright green fluorescence $\left(\lambda_{\mathrm{em}}=503 \mathrm{~nm}, \Phi=0.95\right.$, Figure 1a). Treatment of RF1 with a variety of mild reductants, including tris(2-carboxyethyl)phosphine (TCEP), sodium dithionite, or $\mathrm{NaBH}_{4}$, proceeds smoothly to generate the reduced RF1 probe, which possesses no absorption features in the visible region and is non-fluorescent with 490-nm excitation. For example, treatment of RF1 with 5 equiv of TCEP results in a $>50$-fold fluorescence decrease for the dye (Figure 1a). The sensor can be re-oxidized by air or hydrogen peroxide to restore its fluorescent state, and the reversible oxidation-reduction cycle can be repeated at least 10 times with no loss of dynamic range for the dye. Figure $1 \mathrm{~b}$ shows representative kinetics traces for three reversible oxidation-reduction cycles mediated by $\mathrm{H}_{2} \mathrm{O}_{2}$ and TCEP. Reduction of RF1 by 5 equiv of TCEP occurs promptly upon mixing, whereas oxidation proceeds more slowly. The observed rate constant for $\mathrm{RF} 1$ re-oxidation by $\mathrm{H}_{2} \mathrm{O}_{2}$ under pseudo first-order conditions is $k_{\text {obs }}=4.0(1.1) \times 10^{-2} \mathrm{~s}^{-1}$. The dual colorimetric/fluorimetric response of RF1 suggests that a simple photoinduced electron transfer mechanism is not operable in this redox cycle. Instead, we propose that reduction of RF1 generates a dihydrofluorescein species from the disulfide through reduction, internal charge transfer, and protonation. Along these lines, the ${ }^{1} \mathrm{H}$ NMR of reduced RF1 shows a new peak at $5.16 \mathrm{ppm}$, consistent with a methine-type proton on the quaternary center of a dihydrofluorescein structure. The reversible response of small molecule RF1 is complementary to fiber optic probes for oxygen and other redox-active analytes. ${ }^{17}$

With spectroscopic results showing the redox sensitivity and reversibility of RF1 in hand, we next tested the ability of this new chemical tool to image reversible redox cycles in living cells. As initial experiments revealed that RF1 is not membrane permeable, we prepared the acetoxymethyl (AM) ester derivative for live-cell labeling. Live HEK 293 cells loaded with 5 $\mu \mathrm{M}$ RF1-AM for $30 \mathrm{~min}$ at $37^{\circ} \mathrm{C}$ show faint fluorescence, consistent with entry of the probe into the cells and reduction by the cytosolic environment (Figure 2a). Washing and treatment of the same RF1-loaded cells with $\mathrm{H}_{2} \mathrm{O}_{2}$ results in a marked increase in intracellular fluorescence within 5-10 min as the probe senses oxidative stress (Figure 2b). After 5-10 more $\mathrm{min}$, the observed intracellular fluorescence decreases to baseline levels as the native reducing environment of the cells is restored (Figure 2c). Control images on other fields of cells in the same experiment show that the lack of fluorescence is not due to RF1 loss or photobleaching. To further demonstrate that low levels of intracellular fluorescence in panel $2 \mathrm{c}$ are not due to photobleaching or loss of dye, addition of a second aliquot of $\mathrm{H}_{2} \mathrm{O}_{2}$ oxidant results in another burst of oxidative stress and increase in intracellular fluorescence (Figure 2d), which subsides again after an additional 5-10 min due to restoration of the reducing cellular environment (Figure 2e). Finally, brightfield transmission images confirm that the cells are still viable after these multiple redox cycling events (Figure 2f). Taken together, the data show that RF1 can be loaded into living cells and report multiple oxidation-reduction cycles by a reversible fluorescence response. The turn-on dynamic range for RF1 oxidation in cells is less than in the spectroscopic experiments, presumably due to the reducing environment of the cell.

In summary, we have presented the synthesis, characterization, and live-cell imaging applications of RF1, a new redox-sensitive optical sensor for monitoring reversible oxidation and reduction events in living systems. RF1 features a reversible change in fluorescence upon oxidation or reduction, a dual colorimetric/fluorimetric response, and visible wavelength excitation and emission profiles. Moreover, the sensor is capable of visualizing multiple cycles of oxidative stress and reductive repair in living cells. Experiments are underway to utilize RF1 
and related chemical tools to study the production, propagation, and termination of oxidative signals in biological systems.

\section{Supplementary Material}

Refer to Web version on PubMed Central for supplementary material.

\section{Acknowledgment}

We thank the University of California, Berkeley, the Dreyfus Foundation, the Beckman Foundation, the American Federation for Aging Research, the National Science Foundation (CAREER Award CHE-0548245), and the National Institute of General Medical Sciences (NIH GM 79465) for research support. E.W.M. was supported by a Chemical Biology Interface Training Grant from the NIH (T32 GM066698) and a Stauffer fellowship, and S.X.B. was funded by a summer undergraduate research fellowship from the UC Berkeley Chemical Biology program. Confocal fluorescence images were acquired at the Molecular Imaging Center at UC Berkeley. We thank Ann Fischer at the UC Berkeley Tissue Culture Facility for expert technical assistance.

\section{References}

1. Finkel T. Curr. Opin. Chem. Biol 2003;15:247-254.

2. Lu T, Pan Y, Kao S-Y, Li C, Kohane I, Chan J, Yankner BA. Nature 2004;429:883-891. [PubMed: 15190254]

3. Balaban RS, Nemoto S, Finkel T. Cell 2005;120:483-495. [PubMed: 15734681]

4. Rhee SG. Science 2006;312:1882-1883. [PubMed: 16809515] and references therein

5. Stone JR, Yang S. Antiox. Redox Signal 2006;8:243-270.

6. Lee J-W, Helmann JD. Nature 2006;440:363-367. [PubMed: 16541078]

7. Tonks NK. Cell 2005;121:667-670. [PubMed: 15935753]

8. Stadtman ER, Van Remmen H, Richardson A, Wehr NB, Levine RL. Biochim. Biophys. Acta 2005;1703:135-140. [PubMed: 15680221]

9. Hempel SL, Buettner GR, O'Malley YQ, Wessels DA, Flaherty DM. Free Rad. Biol. Med 1999;27:146159. [PubMed: 10443931]

10. Negre-Salvayre A, Augé N, Duval C, Robbesyn F, Thiers J-C, Nazzal D, Benoist H, Salvayre R. Meth. Enzymol 2002;352:62-71. [PubMed: 12125377]

11. Haugland, RP. The Handbook: A Guide to Fluorescent Probes and Labeling Technologies. 10th Ed.. Molecular Probes/Invitrogen; 2005.

12. Østergaard H, Henriksen A, Hansen FG, Winther JR. EMBO J 2001;20:5853-5862. [PubMed: 11689426]

13. Hanson GT, Aggeler R, Oglesbee D, Cannon M, Capaldi RA, Tsien RY, Remington SJ. J. Biol. Chem 2004;279:13044-13053. [PubMed: 14722062]

14. Lee K, Dzubeck V, Latshaw L, Schneider JP. J. Am. Chem. Soc 2004;126:13616-13617. [PubMed: 15493909]

15. Cline DJ, Thorpe C, Schneider JP. Anal. Biochem 2004;325:144-150. [PubMed: 14715295]

16. Ashe AJ III, Kampf JW, Savla PM. Heteroatom Chem 1994;5:113-119.

17. Wolfbeis OS. Anal. Chem 2006;78:3859-3874. [PubMed: 16771528] 

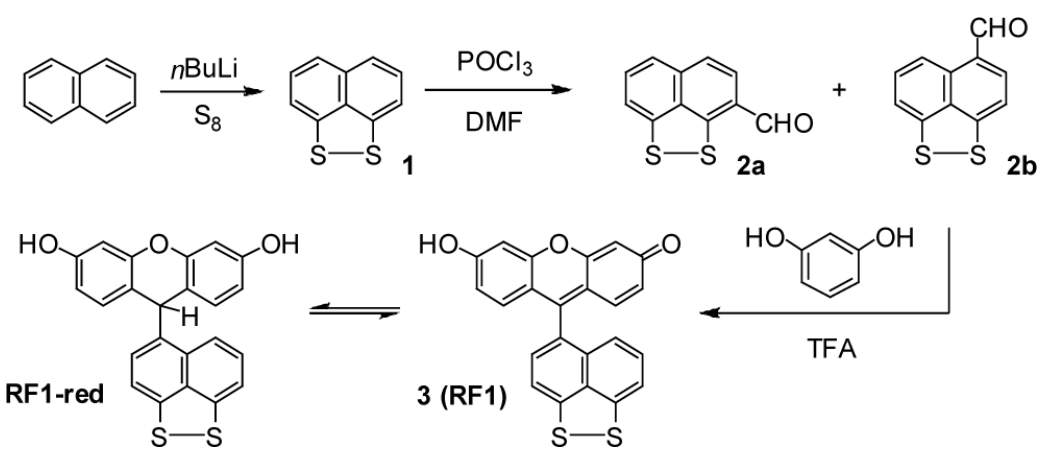

Scheme 1.

Synthesis and Redox Cycling of Redoxfluor-1 (RF1) 

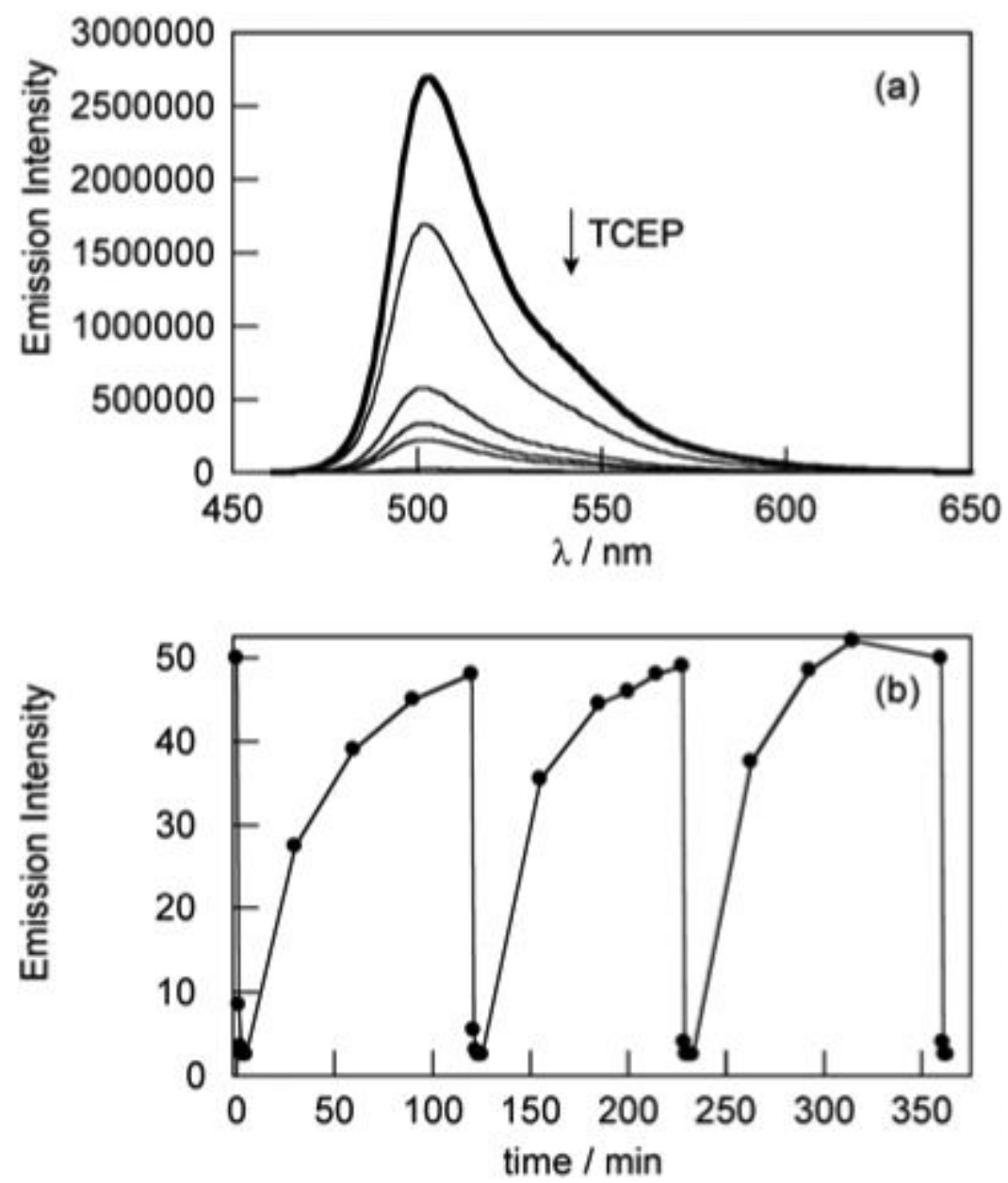

Figure 1.

(a) Fluorescence responses of $5 \mu \mathrm{M}$ RF1 to tris(2-carboxyethyl)phosphine (TCEP). Spectra are shown for $0,1,2,3,4$, and 5 equiv of added TCEP. Spectra were acquired at $25^{\circ} \mathrm{C}$ in 20 $\mathrm{mM}$ HEPES, $\mathrm{pH} 7$, with excitation at $450 \mathrm{~nm}$. (b) Fluorescence responses of RF1 to cycles of oxidation and reduction at $37^{\circ} \mathrm{C}$. A solution of $5 \mu \mathrm{M}$ RF1 was treated with $25 \mu \mathrm{M}$ TCEP reductant and then oxidized with $100 \mu \mathrm{M} \mathrm{H}_{2} \mathrm{O}_{2}$. When fluorescence returned to starting levels, another $25 \mu \mathrm{M}$ aliquot of TCEP was added and the redox cycle was repeated 2 more times without loss of fluorescence. Spectra were acquired in $20 \mathrm{mM}$ HEPES, pH 7, with excitation at $495 \mathrm{~nm}$. Emission intensity was measured at the $\lambda_{\max }$ of $503 \mathrm{~nm}$. 

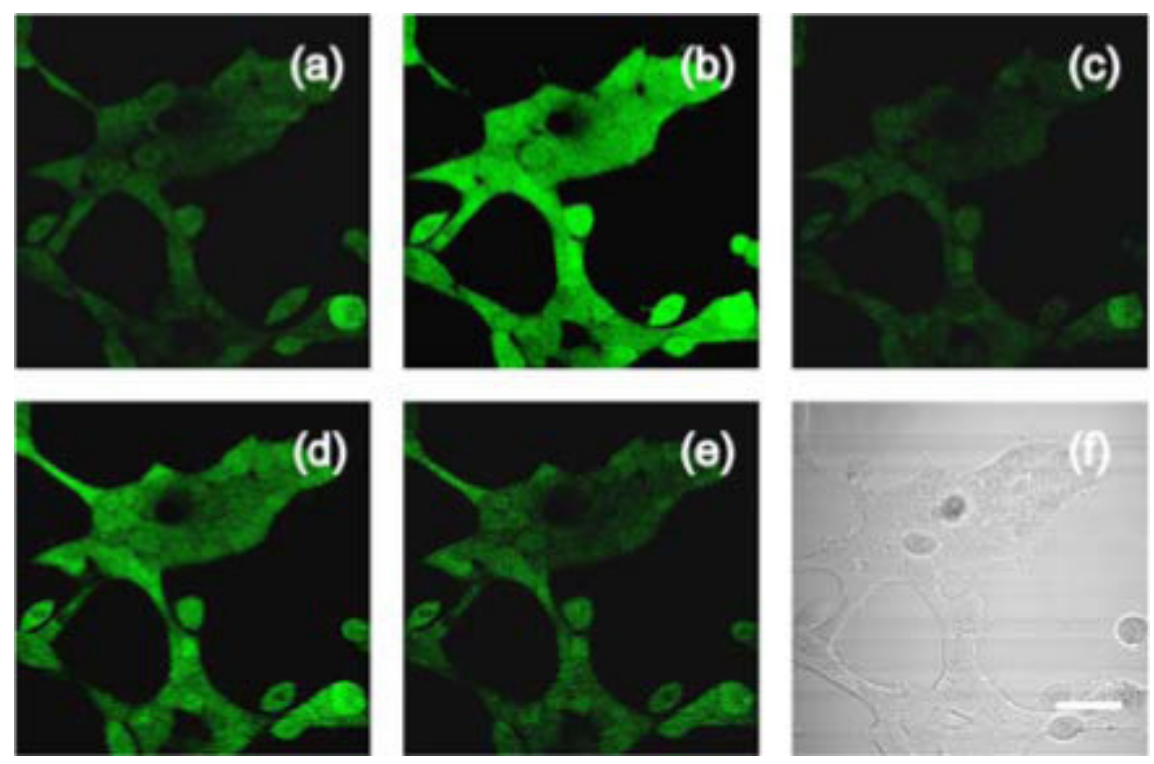

Figure 2. Live-cell imaging of oxidative stress/repair by confocal microscopy at $37{ }^{\circ} \mathrm{C}$

(a) HEK cells loaded with $5 \mu \mathrm{M}$ RF1-AM for 30 min. (b) RF1-loaded HEK cells treated with $100 \mu \mathrm{M} \mathrm{H}_{2} \mathrm{O}_{2}$ for 9 min. (c) $\mathrm{RF} 1$-loaded, $\mathrm{H}_{2} \mathrm{O}_{2}$-treated cells in panel $2 \mathrm{~b}$ after an additional 6 min. (d) Cells exposed to a second dose of $\mathrm{H}_{2} \mathrm{O}_{2}(100 \mu \mathrm{M})$ for an additional 9 min. (e) Cells in panel $2 \mathrm{~d}$ after an additional 6 min. (f) Brightfield image of live HEK cells in panels 2a-2e, confirming their viability throughout the experiment. Scale bar $=10 \mu \mathrm{m}$. 